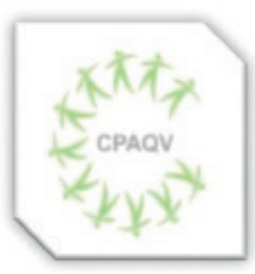

ISSN: 2178-7514
ARTIGO ORIGINAL

\section{A PARTICIPAÇÃO DE ADOLESCENTES EM ESPORTES RADICAIS E DE CONTATO COM A NATUREZA EM PORTO VELHO}

The participation of adolescents in radical sports and in contact with nature in Porto Velho

Ageu N. Coelho ${ }^{14}$; Bruno F. Antunez ${ }^{12}$; Onezímo G. Silva ${ }^{13}$; Mila A. M. Rodrigues ${ }^{13}$; Luís F. Silio ${ }^{11}$; José Ricardo L. Oliveira ${ }^{1}$; Heleise F. R. Oliveira ${ }^{3}$; Adriano A. Pereira ${ }^{1}$; Bráulio N. Lima ${ }^{1,4}$; Carlos H. P. Fileni ${ }^{1,5,6}$; Gustavo C. Martins ${ }^{5,7,8}$; Leandro B. Camargo ${ }^{1,9}$; Alexandre F. Carvalho ${ }^{1,10}$; Ricardo P. Passos ${ }^{1,2}$; Guanis B. Vilela Junior ${ }^{1}$; Célio J. Borges ${ }^{14}$

Vol. 12 | N'. 1| Ano 2021

\title{
RESUMO
}

Os esportes radicais também são conhecidos como esporte de aventura, pois em alguns casos oferece um grande risco devido às condições extremas nos quais são praticados, onde é preciso treinamento, conhecimento, bom equipamento e boa condição física. Surgiram no fim dos anos 80 para o início dos anos 90, tem como principal característica o aumento de adrenalina. Objetivo: Investigar sobre as práticas de esportes radicais e em contato com a natureza por adolescentes na cidade de Porto Velho no período de 2013 e 2019. Métodos: Trata-se de um estudo de campo de caráter descritivo e comparativo. A coleta de dados foi realiza em duas etapas através de um questionário contendo três perguntas fechadas. A primeira foi realizada no mês de abril de 2013, com 56 participantes de ambos os sexos, onde a coleta de dados deu-se em três lugares de Porto Velho, sendo eles o Parque Circuito, Praça do Skate Parque e Praça Aluísio Ferreira, onde ocorriam práticas de esportes radicais. A segunda etapa foi realizada em maio de 2019 com 60 participantes de ambos os sexos, onde a coleta de dados deu-se em três lugares de Porto Velho, sendo Parque da Cidade, Espaço Alternativo e Parque Natural de Porto Velho. O propósito do estudo foi de atualizar os dados e de forma comparativa verificar quais mudanças acorreu no período de seis anos na pratica do esporte radical com adolescentes em Porto Velho. Resultados: Na primeira pesquisa 50\% dos adolescentes tinham como skate o esporte mais praticado, e que $37 \%$ praticam esportes radicais três vezes por semana, que 45\% praticam com o objetivo de melhor qualidade de vida. Na segunda pesquisa, observou-se que o esporte de aventura mais praticado foi o paintball / airsoft com 53\% da preferência, referente ao interesse de praticar o esporte, $70 \%$ dos participantes praticam por prazer e satisfação, sobre a frequência semanal a pesquisa mostra que $70 \%$ dos respondentes praticam uma vez na semana. Conclusão: Conclui-se que os participantes aumentaram a sua participação em outros esportes de aventura em relação aos dados coletados em 2013, a frequência de pratica diminuiu, porém aumentou o prazer pela prática desses esportes.

Palavras-chave: Esportes de Aventura, Adolescentes, Escola, Frequência

\section{ABSTRACT}

Extreme sports are also known as adventure sports, as in some cases they offer a great risk due to the extreme conditions in which they are practiced, where training, knowledge, good equipment and good physical condition are needed. They emerged in the late 1980s to the early 1990s, their main characteristic being the increase in adrenaline. Objective: To investigate the practices of extreme sports and in contact with nature by adolescents in the city of Porto Velho in the period of 2013 and 2019. Methods: This is a descriptive and comparative field study. Data collection was carried out in two stages through a questionnaire containing three closed questions. The first took place in April 2013, with 56 participants of both sexes, where data collection took place in three places in Porto Velho, namely Park Circuit, Skate Square Park and Aluísio Ferreira Square, where extreme sports practices took place. The second stage was held in May 2019 with 60 participants of both sexes, where the data collection took place in three places in Porto Velho, being city's park, Alternative Space and Porto Velho Natural Park. The purpose of the study was to update the data and comparatively verify what changes occurred in the period of six years in the practice of extreme sport with adolescents in Porto Velho. Results: In the first survey, 50\% of adolescents had the most practiced sport as a skateboard, and that 37\% practiced extreme sports three times a week, while $45 \%$ practiced with the objective of better quality of life. In the second survey, it was observed that the most practiced adventure sport was paintball / airsoft with $53 \%$ of preference, referring to the interest in practicing the sport, $70 \%$ of the participants practice for pleasure and satisfaction, on the weekly frequency the research shows that $70 \%$ of respondents practice once a week. Conclusion: It is concluded that the participants increased their participation in other adventure sports in relation to the data collected in 2013, the frequency of practice decreased, but the pleasure in the practice of these sports increased.

Keywords: Extreme Sports, Teens, School, Frequency

Autor de correspondência

Ageu neves coelho

ageunevescoelho1989@gmail.com

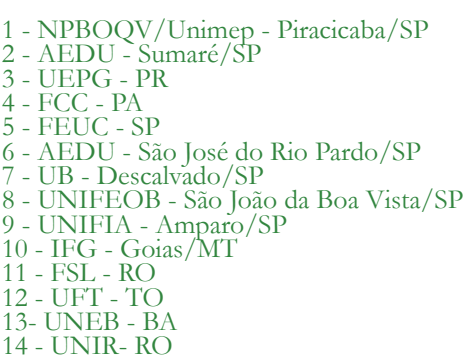

DOI: $10.36692 / \mathrm{v} 13 \mathrm{n} 1-3$ 


\section{INTRODUÇÃO}

A definição de esporte de aventura, também conhecido como esporte da natureza surgiu no final da década de 1980 e início da década de 1990, o termo foi usado para designar como esporte de adultos, tais como: como paintball, skydiving, surfe, alpinismo, montanhismo, paraquedismo, hang gliding, bungee jumping, trekking, ciclismo de montanha, skate e moutain bike praticados apenas por uma minoria, que rapidamente veio e se popularizar ${ }^{(1)}$.

Como destaca Fernandes ${ }^{(2)}$, por sua abrangência os Esportes Radicais podem ser considerados como conjunto de práticas corporais diferenciados por sua aproximação com a natureza ou interação com obstáculos urbanos, e por expressar valores que contestam os padrões antes estabelecidos.

De acordo com Bruhns e Marinho(3) os esportes de aventura e natureza apresentam atualmente três âmbitos de atuação: o turístico recreativo, o de rendimento / competição e o educativo-pedagógico. O terceiro âmbito referese à progressiva incorporação dessas atividades no entorno educativo.

Conhecidos também como esportes de ação, estas práticas esportivas se diferenciam das demais por apresentarem maior grau de risco físico, dado às condições de altura, velocidade ou outras variantes em que são praticados ${ }^{(4)}$. Por contarem com um risco maior, estes esportes são considerados mais emocionantes, exigindo um maior esforço físico e maior controle emocional.

Os esportes radicais/ aventura estão se difundido muito, e no seu contexto vem sendo praticado também por adolescentes, pois é necessário ter um bom condicionamento físico. Segundo Uvinha ${ }^{(5)}$, "a palavra radical geralmente sugere dois entendimentos: extremismo, quando aplicada ao campo da política; raiz, quando se busca a origem de algo, frequentemente utilizada, por exemplo, no campo da filosofia”.

Segundo Tahara \& Scwartz(6), "A busca pela aventura, pelo desconhecido, longe dos padrões urbanos, tem se mostrado mais frequente ultimamente, quando se percebe o aumento de vivências naturais, presente nas atividades físicas de aventura em contato direto com o meio ambiente natural, no sentido de buscar condições favoráveis à possibilidade de imprimir mais qualidade à vida".

Atualmente um aspecto que tem chamado à atenção de muitos jovens e adolescentes, são as atividades de risco conhecido como Esportes Radicais, nas quais são praticadas como lazer, esporte ou educação, em que o movimento é o elemento central dessa cultura ${ }^{(7,8)}$.

O objetivo do estudo é investigar práticas de esportes radicais e em contato com a natureza por adolescentes na cidade de Porto Velho no período de 2013 e 2019.

\section{MÉTODOS}

\section{Amostra}

Trata-se de um estudo quanti-qualitativa de caráter descritivo ${ }^{(9)}$. A pesquisa foi realizada em três praças na cidade de Porto Velho - RO, onde ambas foram selecionadas devido á seus espaços possibilitarem a prática de esportes, sendo que em 2013 foi realizada no Park Circuito, Praça Aluísio Ferreira e Praça do Skate Park e no ano de 2019 realizada no Parque da Cidade, Espaço Alternativo e Parque Natural de Porto Velho.

A pesquisa em sua primeira etapa foi composta de 56 adolescentes, sendo 40 meninos e 16 meninas. Já na segunda etapa foi composta de 60 adolescentes, sendo 40 meninos e 20 meninas, totalizando 116 respondentes que estavam disponíveis no momento, com a faixa etária de 12 a 18 anos, todos praticantes de esportes radicais / aventura.

Todos adolescentes que aceitaram participar da pesquisa, foram orientados e explicado todo o procedimento e que seus responsáveis assinaram o Termo de Consentimento Livre e Esclarecido (TCLE).

\section{Procedimentos Metodológicos}

A pesquisa foi realizada em duas etapas, a primeira foi realizada no mês de março de 2013 onde 56 adolescentes participaram, sendo 16 do 
sexo feminino e 40 masculinos. Os questionários em 2013 foram administrados do dia 15 ao dia 16 de março de 2013, com adolescentes presentes no período vespertino na Praça Aluísio Ferreira, Park Circuito e Praça do Skate Park, situadas na cidade de Porto Velho - Rondônia. Para essa primeira aplicação foram necessários dois dias.

Após abordagem aos adolescentes, foi explicado a cada um o objetivo do presente estudo e sanadas as dúvidas que viessem a ocorrer. Não foi permitida a realização de consultas para responder o questionário e não houve tempo estipulado para responder as questões.

Já a segunda pesquisa foi realizada nos dias 10 e 11 de maio de 2019, neste caso houve uma mudança dos locais que não haviam na pesquisa anterior, tendo sido realizada no parque da cidade, espaço alternativo e Parque Natural de Porto Velho, tendo sido realizado os mesmos procedimentos de abordagem anterior. Nesta segunda obteve-se 60 respondentes, sendo 20 meninas e 40 meninos com idade entre 12 e 17 anos.

Nas duas pesquisas os questionários foram aplicados em um final de semana tendo em vista o maior número de participantes.

As duas pesquisas foram realizadas em dois dias, no primeiro dia foi feito contato com os adolescentes, dadas toda a explicação sobre a pesquisa e sanadas todas as dúvidas que surgiram. Posteriormente foi entregue o TCLE para que os respondentes levassem para seus responsáveis assinar, e foi combinado que no dia seguinte ele seria entregue no mesmo local e horário. Aos adolescentes que retornaram com o TCLE devidamente assinado, foi entregue o questionário e sanada todas as dúvidas para que esses assim respondessem.

\section{Instrumento de Coleta de Dados}

Para coleta de dados, foi utilizado um questionário contendo três perguntas de caráter discursivo, elaborado com base em Tahara e Schwartz ${ }^{(6)}$, sobre a influência, a frequência e as modalidades de esportes radicais praticados por adolescentes.
Usualmente o questionário é a tentativa de conseguir informações sobre práticas e condições atuais, dados demográficos. Às vezes utiliza-se essa técnica para pedir opiniões ou expressões de conhecimentos $^{(10)}$.

\section{Tratamentos Estatísticos}

Os dados foram tabulados e analisados por meio da estatística descritiva. Após a coleta dos dados as frequências de cada resposta foram tabuladas em uma planilha do programa Excel Office $2018^{\circledR}$, sendo os dados apresentados em forma de valores de porcentagens, representados em gráficos para melhor visualização.

\section{RESULTADOS E DISCUSSÃO}

A seguir serão apresentados os resultados da pesquisa, assim como sua análise de acordo com as respostas obtidas em cada pergunta: Segundo suas preferências, quais os principais tipos de atividades de aventura mais praticados? Qual a frequência semanal de assiduidade à prática? Quais seus principais interesses na prática das atividades de aventura na natureza?

$$
\text { Para uma melhor visualização e }
$$
compreensão, os resultados obtidos foram colocados em porcentagem, usando assim um único gráfico para os resultados de cada pergunta dos anos de 2013 e 2019, podendo assim ser feito um melhor comparativo entre os dois resultados.

O gráfico 01 traz referência ao gênero dos participantes das duas pesquisas.

Verifica-se que na primeira pesquisa (2013) obteve-se aproximadamente $72 \%$ de adolescentes do sexo masculino e aproximadamente $28 \%$ do sexo feminino. E na segunda pesquisa (2019) mostra-se que $67 \%$ dos adolescentes participantes são do sexo masculino e $33 \%$ do sexo feminino. Observa-se que em relação à pesquisa anterior houve um aumento de $3 \%$ da população feminina participante, logo uma pequena diminuição de $3 \%$ dos adolescentes do sexo masculino. 
Gráfico 1: Participantes separados por GÊNERO.

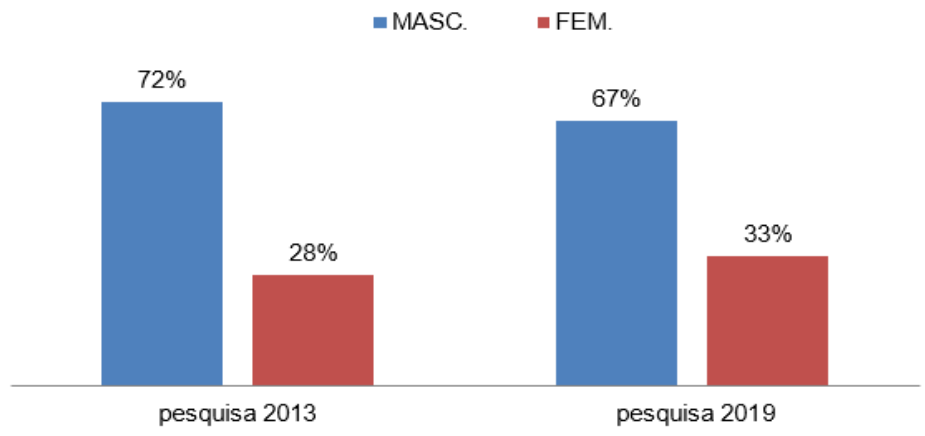

Gráfico 01: Referente ao gênero, 2013 e 2019.

O gráfico 02 faz-se referência a primeira pergunta do questionário, que é: Segundo suas preferências, quais os principais tipos de atividades de aventura mais praticados?

Trata-se da preferência pelo esporte radical e qual modalidade os participantes pratica com frequência.

Pode-se observar que em 2013 a preferência sobre o skate foi de $50 \%$, seguido da trilha $27 \%$, moutain bike $17 \%$ e $8 \%$ outros. Já na segunda pesquisa (2019) observa-se que 53\% dos adolescentes tem a preferência pela prática do airsoft / paint ball, 25\% por motainbike, $12 \%$ tem preferência pela prática do skate e 10\% escolheram entre trilha e esportes de aventura.

Nesta segunda pesquisa a opção outros foi preenchida pelos participantes somente com a opção de airsoft e paint ball. Verifica-se que a grande mudança que ouve entre as duas pesquisas, quando em 2013 o skate era o esporte de aventura mais praticado, já em 2019 o airsoft/ paint ball se destaca com o maior índice.

Os achados neste estudo se diferem com a pesquisa realizada no Rio de Janeiro por Triani et al., ${ }^{(11)}$ onde foi possível afirmar que os esportes de aventura mais praticados são voo livre (81\%), kitesurf $(7 \%), \operatorname{surf}(5 \%)$, trilha $(5 \%)$, rapel $(2 \%)$ e escalada $(1 \%)$.

Gráfico 2- Segundo suas PREFERÊNCIAS, quais os principais tipos de atividades de aventura mais praticados?

=SKATE $=$ TRILHA $\cong$ MOTAINBIKE $\cong$ OUTROS

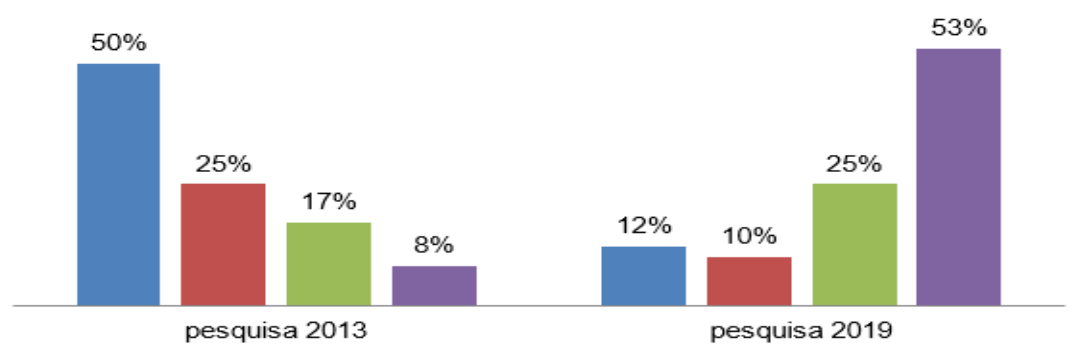

Gráfico 02: Referente à Preferência, 2013 e 2019. 
O gráfico 03 expõe a seguinte questão: Qual a frequência semanal de assiduidade à prática?

Foi abordado a frequência de pratica de tal modalidade durante a semana.

Tendo como resultado em 2013, 37\% praticavam atividades de aventura ao menos três vezes na semana, $33 \%$ praticavam duas vezes na semana e 30\% apenas uma única vez na semana.

Em relação a frequência que é realizada os esportes de aventura, o gráfico referente a
2019 mostra que 70\% realizam apenas uma vez por semana, $20 \%$ duas vezes na semana e apenas $10 \%$ pratica três vezes na semana.

Notasse um equilíbrio na pratica semanal de esportes de aventura em 2013. Logo em 2019 $70 \%$ dos adolescentes praticam uma vez por semana o esporte de aventura. Estes resultados estão em projeção para os achados de Tahara \& Schwartz ${ }^{(6)}$, onde a frequência de prática dos participantes eram de 3 vezes aos finais de semana durante o mês.

Gráfico 3 - Qual a FREQUÊNCIA semanal de assiduidade à prática?

$\because 1 \mathrm{VZ}$ POR SEMANA $\approx 2 \mathrm{VZ}$ POR SEMANA $\approx 3 \mathrm{VZ}$ POR SEMANA



Gráfico 03: Referente à Frequência, 2013 e 2019.

O gráfico 04 tem como objetivo averiguar:

Quais seus principais interesses na prática das atividades de aventura na natureza?

Saber o que motivou esses adolescentes a praticar a modalidade específica de esporte de aventura, tendo em vista buscar se ouve influencia, entre outras motivações.

Podemos obter o seguinte resultado em 2013: 45\% alegou que praticam por motivo de ter uma melhor qualidade de vida, 18\% alega praticar apenas em busca de novas emoções, $21 \%$ faz a pratica do esporte de aventura por puro prazer e satisfação e 16\% buscam fins estéticos.

O resultado de 2019 mostra que, $70 \%$ dos adolescentes praticam o esporte de aventura pelo prazer e satisfação, enquanto 18\% alegam que fazem a pratica em busca de novas emoções, 7\% estão em busca de uma melhor qualidade de vida e $5 \%$ buscam apenas fins estéticos.

Observa-se que em 2013 o objetivo da prática era uma melhor percepção de qualidade de vida com 45\%, em 2019 a maioria dos 
adolescentes alegaram que praticam com o aderido à prática do Mountain Bike no interior de objetivo de prazer e satisfação pelo esporte com São Paulo, concluindo que, o Mountain Bike traz $70 \%$. melhorias e mudanças significativas nos aspectos

No estudo de Biazotti et al., ${ }^{(12)}$ buscou físicos, cognitivos e psicossociais, corroborando analisar e identificar o motivo das pessoas ter assim com os achados neste estudo.

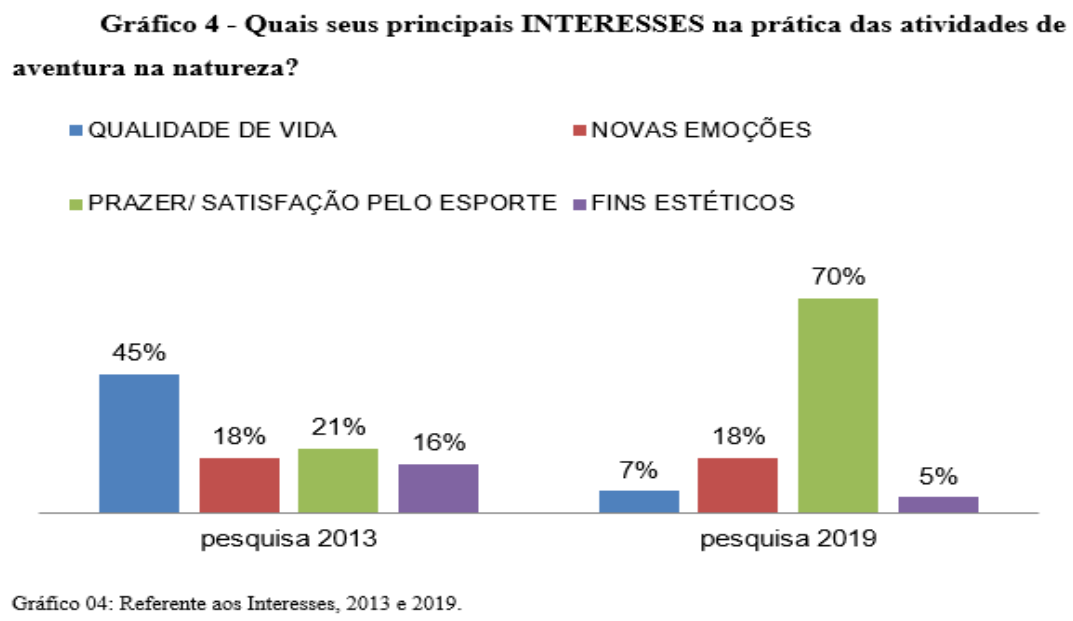

Diante do resultado da pesquisa que foi realizada no ano de 2013, podemos observa que a maior parte dos adolescentes pertence ao sexo masculino, sendo no total $72 \%$, podemos observa também que a modalidade mais praticada e a de skate, com $50 \%$ da preferência. A frequência que as modalidades eram praticadas, nos mostra que ouve pouca diferenciação, sendo que a diferença da primeira para a última ficou apenas com 7\% mostra também que se levar em consideração que uma a duas vezes por semana e o número baixo de frequência para se praticar um esporte.

Quando chegamos à parte do interesse verificamos que a melhor qualidade de vida e a intenção principal dos praticantes, ou seja, em busca de uma vida saudável com mais saúde.

Ao fazermos uma breve comparação com os dados obtidos da pesquisa no ano de
2019 podemos notar que ouve um aumento do número de praticante do sexo feminino, que em 2013 era de $28 \%$ passou a ser 33\% em 2019.

A respeito da frequência com que é praticado o esporte de aventura, notamos que em 2013 a maioria dos participantes praticava três vezes semanal, sendo estes $37 \%$ da amostra e que em 2019 a grande maioria praticava apenas uma vez semanais sendo $70 \%$ dos adolescentes pesquisados.

Quando chegamos à Parte do interesse desses adolescentes, em 2013 45\% alegou que praticava os esportes de aventura para ter uma melhor qualidade de vida, já em 2019 70\% da amostra disse que pratica apenas pelo prazer e satisfação.

O estudo mostrou que em 2013, 50\% dos participantes tinham o skate como a principal 
prática, já em 2019 teve uma reviravolta, pois teve com 70\% o airsoft/paint ball como o esporte de aventura mais praticado, sendo que nos anos de 2013 o mesmo não foi citado como opção de esporte de aventura.

Podemos concluir que a Cidade de Porto Velho se desenvolveu e expandiu, trazendo assim novas opções de esportes para os habitantes, e que a cidade ainda está em desenvolvimento.

\section{CONSIDERAÇÕES FINAIS}

Apesar de ter se expandido no mundo todo, no Brasil, na região norte, o esporte de aventura vem crescendo não na mesma proporção que a nível mundial, mais está se enraizando aos poucos no nosso estado. Com base nos resultados do estudo, o skate (em 2013) e o airsoft / paintball (2019) são as atividades de aventura mais praticadas. A frequência semanal mais citada foi de três vezes por semana para o skate e uma vez para airsoft / paintball, concorrer na tentativa de melhoria dos níveis de qualidade de vida, além da satisfação e prazer pelo esporte, foram os principais interesses do público alvo da pesquisa, quando o mesmo realiza atividades em contato direto com o meio ambiente natural.

Tais práticas, através da experiência significativa por elas proporcionada, tendem a despertar em seus adeptos sentimentos contemplativos e emotivos, filtrado em desejos e valores, fundamentados na ética de profundo respeito perante a biodiversidade da natureza.
Torna-se possível, com base nos resultados do estudo, reconhecer, de maneira mais facilitada, as potencialidades e abrangência dos níveis qualitativos que a interação humana com o meio natural pode representar.

Torna-se, desta forma, bastante instigante aprofundar as reflexões sobre estas temáticas, a fim de ampliar as discussões e conhecer melhor o potencial do universo focalizado, no que tange às perspectivas de melhoria dos aspectos qualitativos da existência.

Este trabalho reforça a importância do esporte de aventura na vida do adolescente e nas aulas de educação física, mostra a importância do Educador Físico na Escola e a Grande responsabilidade e o valor que tem. Pois os movimentos e habilidades que são adquiridos com a prática do esporte de aventura, contribui de maneira significativa para evoluir as habilidades e capacidades motoras.

\section{REFERÊNCIAS}

1.Auricchio JR. Escalada na Educação Física Escolar. Orientação adequada para a prática segura. Lecturas: educación física y deportes, Buenos Aires. 2009;14(139). 2.de Cassia Fernandes R. ESPORTES RADICAIS: REFERÊNCIAS PARA UM ESTUDO ACADÊMICO RADICAL SPORTS. Conexões. 1998;1(1):95-.

3.Bruhns HT, Marinho A. Viagens, lazer e esporte: Editora Manole Ltda; 2006.

4.SCHWARTZ GM. Emoção, aventura e risco-a dinâmica metafórica dos novos estilos. Lazer e estilo de vida Santa Cruz do Sul: EDUNISC. 2002:139-68.

5.Uvinha RR. Juventude, lazer e esportes radicais: Editora Manole Ltda; 2001.

6.Tahara AK, Schwartz GM. Atividades de aventura na natureza: investindo na qualidade de vida. Lecturas. 2003;8:58.

7.Pereira DW, Armbrust I, Ricardo DP. Esportes radicais, de aventura e ação: conceitos, classificações e características. Corpoconsciência. 2010;12(1):18-34. 
8.Marinho A, Schwartz GM. Atividades de aventura como conteúdo da educação física: reflexões sobre seu valor educativo. Revista Digital, Buenos Aires. 2005;10(88):1-7.

9.Fontelles MJ, Simões MG, Farias SH, Fontelles RGS. Metodologia da pesquisa científica: diretrizes para a elaboração de um protocolo de pesquisa. Revista paraense de medicina. 2009;23(3):1-8.

10.Thomas JR, Nelson JK, Silverman SJ. Métodos de pesquisa em atividade física: Artmed Editora; 2009.

11.da Silva Triani F, Sampaio BHR, de Castro LM, da Paixão JA. Esportes de aventura praticados na Barra da Tijuca e São Conrado, RJ: um levantamento das modalidades e formação do instrutor. Motrivivência. 2020;32(61):01-15.

12.da Silva Biazotti P, da Silva FH, Pinheiro AM, de Souza Vespasiano B. Fatores de adesão da prática do Mountain Bike. RBPFEX-Revista Brasileira de Prescrição e Fisiologia do Exercício. 2016;10(62):806-14.

OBSERVAÇÃO: Os autores declaram não existir conflitos de interesse de qualquer natureza. 